\title{
The TORC1 inhibitors Nprl2 and Nprl3 mediate an adaptive response to amino-acid starvation in Drosophila
}

\author{
Y Wei ${ }^{1}$ and MA Lilly ${ }^{*, 1}$
}

Target of rapamycin complex 1 (TORC1) is a master regulator of metabolism in eukaryotes that integrates information from multiple upstream signaling pathways. In yeast, the Nitrogen permease regulators 2 and 3 (Npr2 and Npr3) mediate an essential response to amino-acid limitation upstream of TORC1. In mammals, the Npr2 ortholog, Nprl2, is a putative tumor suppressor gene that inhibits cell growth and enhances sensitivity to numerous anticancer drugs including cisplatin. However, the precise role of Nprl2 and Nprl3 in the regulation of metabolism in metazoans remains poorly defined. Here we demonstrate that the central importance of Nprl2 and Nprl3 in the response to amino-acid starvation has been conserved from single celled to multicellular animals. We find that in Drosophila Nprl2 and Nprl3 physically interact and are targeted to lysosomes and autolysosomes. Using oogenesis as a model system, we show that Nprl2 and Nprl3 inhibit TORC1 signaling in the female germline in response to amino-acid starvation. Moreover, the inhibition TORC 1 by Nprl2/3 is critical to the preservation of female fertility during times of protein scarcity. In young egg chambers the failure to downregulate TORC1 in response to amino-acid limitation triggers apoptosis. Thus, our data suggest the presence of a metabolic checkpoint that initiates a cell death program when TORC1 activity remains inappropriately high during periods of amino-acid and/or nutrient scarcity in oogenesis. Finally, we demonstrate that Nprl2/3 work in concert with the TORC1 inhibitors Tsc1/2 to fine tune TORC1 activity during oogenesis and that Tsc1 is a critical downstream effector of Akt1 in the female germline.

Cell Death and Differentiation (2014) 21, 1460-1468; doi:10.1038/cdd.2014.63; published online 2 May 2014

In Drosophila, egg production is an energy intensive process that occurs continuously throughout the lifetime of the female. Thus, to ensure that energy reserves remain sufficient to support the viability of the female and her progeny during times of food scarcity, Drosophila oogenesis is highly sensitive to nutritional inputs. ${ }^{1-3}$ The Drosophila ovary is comprised of approximately 15 ovarioles that contain strings of egg chambers in successively older stages of development. ${ }^{4}$ Each egg chamber contains a 16-cell interconnected germline syncytium comprised of 15 polyploid nurse cells and a single oocyte. Each ovarian cyst is surrounded by a somatically derived monolayer of cells called follicle cells. At the tip of the ovariole lies the germarium that contains both germline and somatic stem cells, allowing for the continuous production of new egg chambers throughout the life of the female. In mid-oogenesis, egg chambers begin the energy intensive process of yolk uptake, known as vitellogenesis, which is followed by a short period of rapid growth in late oogenesis prior to the eggs being laid.

Faced with insufficient protein, the Drosophila ovary initiates a complex series of adaptive responses. ${ }^{2,3,5-8} \mathrm{Egg}$ chambers in mid-oogenesis (stages 8-9), which have begun vitellogenesis, undergo apoptosis as do a fraction of early ovarian cysts before their packing by follicle cells in the germarium. ${ }^{2}$ In contrast, young egg chambers (stages 2-7) remain intact, but sharply reduce their growth rates and rearrange their cytoskeletal network. ${ }^{2,5}$ After shutting down oogenesis during a period of starvation, these young dormant egg chambers can be used to rapidly restart egg production when nutrients are reintroduced. ${ }^{2,5}$ Thus, protecting young egg chambers from the ravages of starvation is important for maximizing fecundity in an environment with uneven food availability.

Recent evidence implicates the Target of Rapamycin Complex 1 (TORC1) in the regulation of growth and nutritional response during Drosophila oogenesis. ${ }^{6,9-11}$ TORC1 contains the nutrient sensitive kinase Target of Rapamycin (TOR) and regulates cell growth and metabolism in response to multiple inputs including amino-acid availability and intracellular energy status. ${ }^{12-16}$ In the presence of sufficient nutrients and appropriate growth signals, the Ragulator and the Rag GTPases target TORC1 to lysosomal membranes where it comes in contact with its activator, the small GTPase Rheb. ${ }^{17-19}$ The downregulation of TORC1 activity under conditions of nutrient stress triggers catabolic metabolism and autophagy. ${ }^{20}$ Autophagy involves the lysosomal degradation of cellular

\footnotetext{
${ }^{1}$ Cell Biology and Metabolism Program, National Institute of Child Health and Human Development, National Institutes of Health, Bethesda, MD, USA *Corresponding author: M Lilly, Cell Biology and Metabolism Program, National Institutes of Health/National Institute of Child Health and Human Development, Building 18T/Room 101, Bethesda, MD 20892, USA. Tel: +1 301435 8428; Fax: +1 301402 0078; E-mail: mlilly@ helix.nih.gov

Abbreviations: Npr2, nitrogen permease regulator 2; Npr3, nitrogen permease regulator 3; Nprl2, nitrogen permease regulator like 2; Nprl3, nitrogen permease regulator like 3; TOR, target of rapamycin; TORC1, target of rapamycin complex 1; GAP, GTPase-activating protein; DEPDC5, DEP domain-containing 5; LAMP1, lysosomal-associated membrane protein 1; Tsc1, tuberous sclerosis 1; raptor, regulatory-associated protein of mTOR; Atg8a, autophagy-specific gene 8a; dsRNA, double-strand RNA; UAS, upstream activating site; shRNA, short-hairpin RNA; PBS, phosphate-buffered saline; FSC, forward light scatter; IP, immunoprecipitate Received 14.1.14; revised 02.4.14; accepted 03.4.14; Edited by E Baehrecke; published online 02.5.14
} 
components to ensure adequate nutrients to support cellular survival during times of nutrient stress. Thus, the ability to downregulate TORC1 activity in response to environmental conditions is critical to cell survival.

In both budding and fission yeast, Npr2 and Npr3 inhibit TORC1 activity in response to amino-acid scarcity. ${ }^{21,22}$ The downregulation of TORC1 by Npr2 and Npr3 is essential to the adaptive response that allows these single-cell eukaryotes to grow on a poor nitrogen source. Recent evidence indicates that Npr2 and Npr3, and their respective mammalian orthologs Nitrogen permease regulator like 2 (Nprl2) and Nitrogen permease regulator like 3 (Nprl3), function as GTPase-activating proteins (GAP) that inhibit TORC1 activity by inactivating the Rag GTPases. ${ }^{23,24}$ As is observed with other genes that inhibit TORC1 kinase activity, $\mathrm{Npr} 2 / \mathrm{Nprl} 2$ is a putative tumor suppressor gene that is deleted in multiple cancers and cancer cell lines. ${ }^{24,25}$ Yet, while Nprl2/3 have been shown to downregulate TORC1 activity in response to amino-acid starvation in tissue culture cells, ${ }^{24}$ the precise physiological requirement for Nprl2 and Nprl3 in the response to nutrient stress remains undefined in metazoans.

Here we demonstrate that in Drosophila Nprl2 and Nprl3 mediate an adaptive response to amino-acid scarcity that is essential to the maintenance of female fertility. We find that in nprl2 and nprl3 germline knockdowns, young egg chambers fail to adapt to amino-acid scarcity and undergo apoptosis. Feeding females the TORC1 inhibitor rapamycin prevents this apoptotic response. Thus, in Drosophila the failure to downregulate TORC1 activity during periods of nutrient stress triggers programmed cell death in early oogenesis. Finally, we demonstrate that the two TORC1 inhibitory complexes Nprl2/ 3 and Tsc1/2 both contribute to the regulation of TORC1 activity in the female germline.

\section{Results}

Nprl2 and Nprl3 physically interact and function as TORC1 inhibitors. Nprl2 and Nprl3 are predicted to form a heterodimer that functions in a trimeric complex with Iml1/ DEPDC5 to inhibit TORC1 activity in response to amino-acid stress. ${ }^{21,23,24,26}$ To confirm the association of Nprl2/CG9104 and Nprl3/CG8783 in Drosophila, we coexpressed the Nprl2$\mathrm{HA}$ and Nprl3-V5 tagged proteins in S2 cells and found that
Nprl2-HA specifically coimmunoprecipitated with Nprl3-V5 (Figure 1a). In order to examine the role of Nprl2/Nprl3 in the regulation of TORC1 activity, we depleted $n p r l 2$ and $n p r l 3$ transcripts in S2 cells using double-strand RNA (dsRNA) and then examined the phosphorylation status of the downstream TORC1 target 4E-BP. When nprl2 or nprl3 was knocked down under conditions of amino-acid depletion, 4E-BP phosphorylation remained inappropriately high relative to controls (Figure 1b). Activation of the TORC1 pathway promotes cell growth and as a consequence increased cell size. ${ }^{27}$ To define further the roles of Nprl2 and Nprl3 in the regulation of TORC1 activity, we examined $\mathrm{S} 2$ cell size using flow cytometry. To avoid bias introduced by alterations in the distribution of cells in each cycle phase, we focused our analysis on cells in G1. In line with their proposed roles in the regulation of TORC1 activity, we found that reducing nprl2 or nprl3 levels increases cell size during amino-acid limitation (Figure 1c). Thus, consistent with a previous report in mammalian cells, ${ }^{24}$ our results demonstrate that Nprl2 and Nprl3 physically interact and are required to inhibit TORC1 activity during amino-acid limitation in Drosophila S2 cells.

Nprl2 and Nprl3 localize to lysosomes and autolysosomes. Recent evidence indicates that lysosomes, the approximate equivalent of the yeast vacuole, are the sites of TORC1 activation. $^{28}$ In mammalian cells DEPDC5, a component of the trimeric DEPDC5, Nprl2, Nprl3 complex localizes to lysosomes under conditions of nutrient stress. ${ }^{24}$ Using live cell imaging of GFP-Nprl2 and Nprl3-mCherry expressed in the female germline, we determined that both Nprl2 and Nprl3 localized to the cytoplasm as well as a to small number of LysoTracker positive structures in the nurse cells and oocyte of fed females (Figures 2a-f). LysoTracker marks acidic compartments and is used to highlight early endosomes/lysosomes and autolysosomes. When females were starved for amino acids, Nprl2 and Nprl3 localized to large puncta that were both LysoTracker and mCherry-Atg8a or GFP-LAMP1 positive. mCherry-Atg8a marks autophagosomes and autolysosomes, while GFP-LAMP1 marks lysosomes and autolysosomes. ${ }^{29,30}$ Taken together, these data strongly suggest that the Nprl2 and Nprl3 proteins localize primarily to autolysosomes under conditions of amino-acid starvation (Figures 2a'-i'). We also noted that a

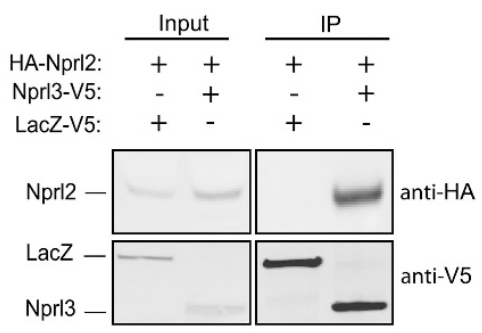

b

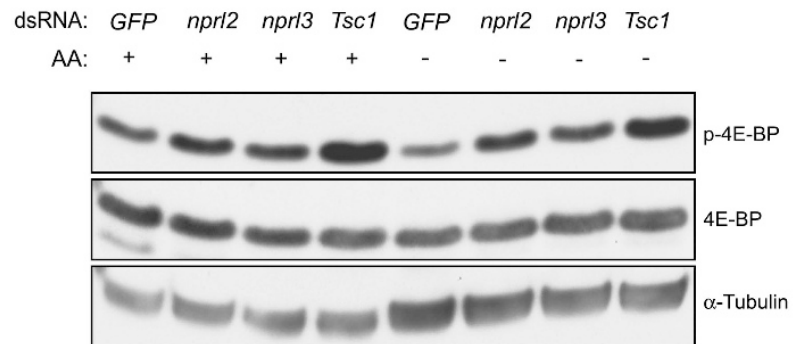

C

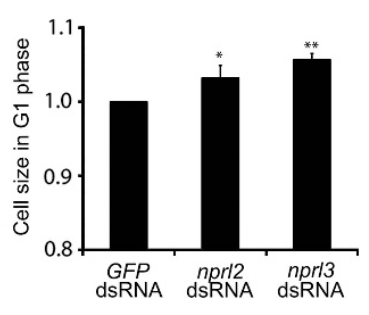

Figure 1 Nprl2 and Nprl3 inhibit TORC1 activity in Drosophila. (a) S2 cells were co-transfected with HA-tagged Nprl2 and V5-tagged Nprl3 or lacZ (control) plasmids. Cells were lysed and immunoprecipitated using an anti-V5 antibody. Cell lysates (inputs) and immunoprecipitates (IP) were detected by western blot using anti-HA and anti-V5 antibodies. (b) S2 cells were treated with GFP, nprl2 or nprl3 dsRNA for 4 days and then cultured in Schneider's medium plus 10\% FBS (AA +) or amino-acid-free Schneider's medium (AA-) for $60 \mathrm{~min}$. The protein levels of $\mathrm{p}-4 \mathrm{E}-\mathrm{BP}$, total $4 \mathrm{E}-\mathrm{BP}$ and $\alpha$-Tubulin were determined by western blot. Similar western Blot results were observed in three independent experiments. (c) S2 cells were treated with GFP, nprl2 or nprl3 dsRNA for 4 days and then cultured in amino-acid-free Schneider's medium plus 10\% FBS for $24 \mathrm{~h}$. Cell size in G1 phase was normalized to GFP dsRNA control. Error bars represent S.D. values from three independent experiments. ${ }^{*} P<0.05 ;{ }^{* *} P<0.01$ 

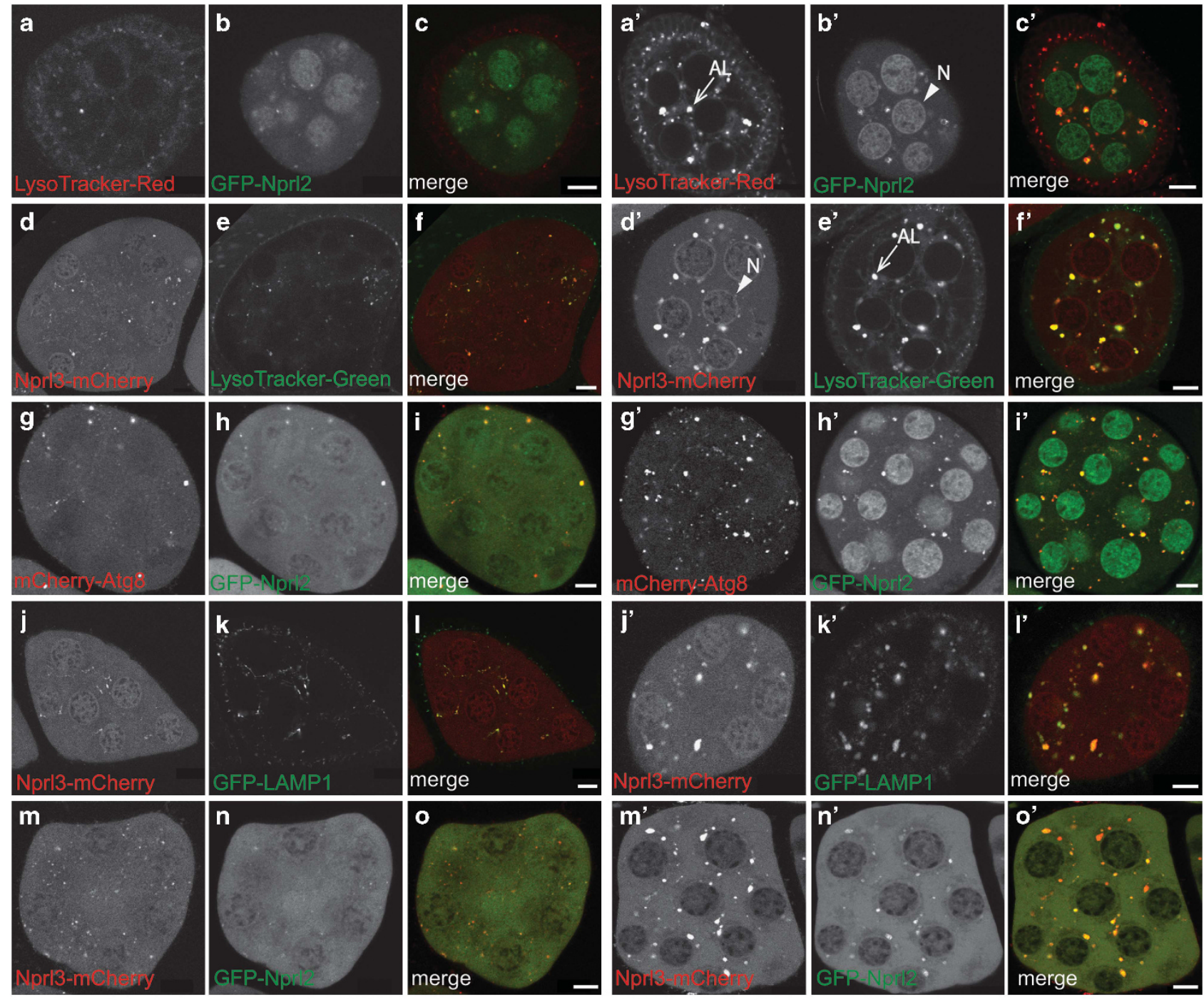

Fed

Starved

Figure 2 Nprl2 and Nprl3 localize to lysosomes, autolysosomes. (a-0 and a'-o') Live cell imaging of Drosophila egg chambers from females cultured on fly media with wet yeast (fed, a-o) or 20\% sucrose (starved, a'-o'). (a-c and a'-c') GFP-Nprl2 co-localizes with LysoTracker Red. (d-f and d'-f') Nprl3-mCherry co-localizes with LysoTracker Green. (g-i and g'-i') GFP-Nprl2 co-localizes with mCherry-Atg8. (j-I and j'-l') Nprl3-mCherry co-localizes with GFP-LAMP1. (m-o and m'-o') GFP-Nprl2 co-localizes with Nprl3-mCherry. Note that Nprl2 and Nprl3 localize to autolysosomes (arrow; AL, autolysosome). In addition, Nprl2 and Nprl3 are found in nuclei (arrowhead; N, nuclei). The MTD-GAL4 driver was used to drive expression of GFP-Nprl2, mCherry-Atg8 and Nprl3-mCherry. GFP-LAMP1 was expressed with a tubulin promoter. Bar, $10 \mu \mathrm{m}$

GFP-Nprl2, and to a lesser extent Nprl3-mCherry, were present in nurse cell and oocyte nuclei with the strongest nuclear staining observed under amino-acid starvation conditions (Figures $2 a$ and d). Finally, when coexpressed in the female germline GFP-Nprl2 and Nprl3-mCherry colocalize under both fed and starved conditions (Figures 2m-0 and m'-o').

Nprl2 and Nprl3 mediate an adaptive response to aminoacid starvation in the female germline. In yeast, mutations in Npr2 or Npr3 result in a diminished ability to grow and survive when amino acids are limited. ${ }^{21,26,31-33}$ To determine whether the basic requirement for $\mathrm{Nprl} 2$ and $\mathrm{Nprl3}$ in mediating a response to amino-acid limitation is conserved in multicellular animals, we examined the role of $\mathrm{Nprl} 2$ and $\mathrm{Nprl3}$ in the Drosophila ovary, a tissue known to exhibit a marked and rapid response to nutrient status. ${ }^{1,2}$ In order to knockdown nprl2 and nprl3 transcript levels, we constructed six transgenic lines that express short-hairpin RNAs (shRNA) that target the nprl2 and nprl3 transcripts (Supplementary Figure S1). These transgenic lines utilize the GAL4-upstream activating site (UAS) to control expression. ${ }^{34}$ When expressed in the female germline using the MTD-GAL4 germline specific driver, we achieved a greater than $90 \%$ reduction of $n p r l 2$ and $n p r / 3$ transcript levels in the ovary for five of the six transgenic lines (Supplementary Figure S1). When nprl2 RNAi and nprl3 RNAi were expressed in the germline of females that had been provided a protein source of wet yeast, the females were fertile with only a small reduction in egg production rates observed in the nprl3 RNAi line (Supplementary Figure S2). Thus, knocking down nprl2 

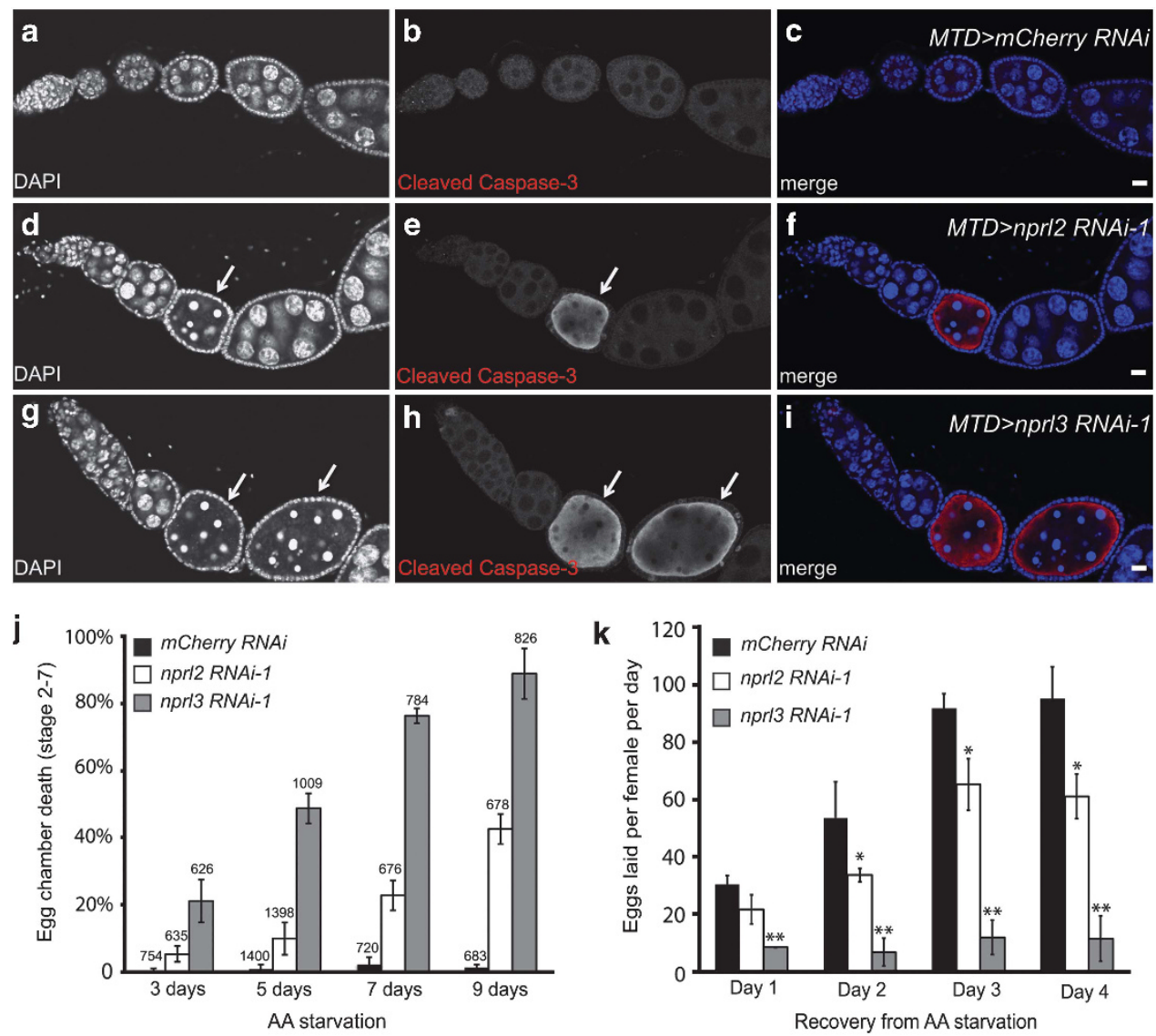

Figure 3 Nprl2 and Nprl3 protect young egg chambers during amino-acid starvation. (a-i) Females were cultured on 20\% sucrose (AA-) for 5 days. (a-c) MTD $>m C h e r r y$ RNAi, (d-f) MTD > nprl2 RNAi-1 and (g-i) MTD > nprl3 RNAi-1 egg chambers, labeled with DAPI (blue) and cleaved Caspase-3 (red). Note that the egg chambers with condensed DNA staining (pyknotic nuclei) were also cleaved Caspase-3 positive (arrows). Bar, $10 \mu \mathrm{m}$. (j) Females were cultured on $20 \%$ sucrose (AA-) for indicated number of days. Percentages of dying egg chambers (stage 2-7) were recorded for each genotype. Error bars represent S.D. of three independent experiments. The numbers above bars represent the number of young egg chambers (stages 2-7) examined. (k) Females were cultured on $20 \%$ sucrose for 7 days and then transferred to fly media containing wet yeast. Data show eggs laid after the cessation of starvation. Error bars represent S.D. of three independent experiments, where each experiment examined greater than 200 ovaries. ${ }^{*} P<0.05 ;{ }^{*} P<0.01$

and nprl3 transcript levels in the female germline had a minimal effect on oogenesis in females that had access to a protein source.

We next examined whether there is a specific requirement for Nprl2 and Nprl3 under conditions of amino-acid scarcity. When females depleted for nprl2 or nprl3 were starved for amino acids, there was a marked increase in the number of degenerating young egg chambers compared with controls (Figures $3 a-i)$. Similar results were observed using three different RNAi targets against $n p r l 2$ and as well as two RNAi targets against nprl3 (Supplementary Table S1). We believe that the different rates of egg chamber death observed using different RNAi targets for nprl2 and nprl3 likely reflect small differences in the efficacy of the RNAi knockdowns. However, currently we can not rule out the possibility that $n p r l 2$ and $n p r l 3$ have slightly different roles in the response to amino-acid starvation and/or that the proteins have different stabilities. For all subsequent experiments, we used the nprl2 RNAi-1 and nprl3 RNAi-1 lines. Notably, in both nprl2 RNAi and nprl3 $R N A i$ ovaries, the degenerating egg chambers contained condensed DNA and were cleaved Caspase 3 positive, indicating that they were undergoing apoptosis. ${ }^{35}$ In protein starved nprl2 RNAiand nprl3 RNAifemales, the percentage of apoptotic young egg chambers increased rapidly with starvation time (Figures 3j). In contrast, in control RNAi females, even after 9 days of protein starvation, only a very small percentage of young egg chambers undergo apoptosis (Figure 3j). These data indicate that Nprl2 and Nprl3 mediate a protective response to amino-acid starvation that is critical to the survival of young egg chambers.

After marked slowing of oogenesis during a period of starvation, Drosophila females can rapidly restart egg production when nutrients are reintroduced. ${ }^{2}$ We examined the restart of oogenesis, after a defined period of amino-acid starvation, in control RNAi, nprl2 RNAi and nprl3 RNAi females by monitoring the number of eggs laid after the reintroduction of a protein source (wet yeast). After 7 days of amino-acid starvation and the near shutdown of oogenesis, the number of eggs laid per control RNAi female rapidly increases after the reintroduction of a protein source (Figure 3k). In contrast, in nprl2 RNAi and nprl3 RNAifemales, the number of eggs laid per female remained low multiple 
days after the reintroduction of protein (Figure 3k). Consistent with this observation, recovering nprl2 RNAi and nprl3 RNAi ovarioles often contained numerous degenerating egg chambers, egg chambers with less than 16 germ line cells, as well as fewer late-stage egg chambers (Supplementary Figure S3). Notably, nprl3 RNAi females fail to recover from a period of amino-acid starvation even 14 days after the reintroduction of protein (data not shown). Thus, in the absence of Nprl3, a brief period protein starvation results in a permanent reduction in fertility. Taken together our data indicate that $\mathrm{Nprl} 2 / 3$ function to preserve female fertility during periods of protein scarcity.

In contrast to young egg chambers, which are resistant to cell death during periods of nutrient limitation, mid-stage egg chambers are shunted into an apoptotic pathway and degenerate. ${ }^{36}$ Surprisingly, in response to amino-acid starvation, we found that mid-stage egg chambers (stages 8-9), from nprl2 RNAi and nprl3 RNAi flies, degenerated in a manner that was indistinguishable from controls, suggesting these genes do not have a role in regulating the response to nutrient stress at this developmental time point (Supplementary Figure S4). In addition, as discussed below, the response of mid-stage egg chambers to the drug rapamycin, a specific inhibitor of TORC1, is indistinguishable from controls in both nprl2 and nprl3 depletions (Supplementary Figures $\mathrm{S} 5 \mathrm{a}-\mathrm{C}$ ). While these results are intriguing, it is important to note that a definitive determination of the role of Nprl2 and Nprl3 in response to nutrient stress in mid-stage egg chambers awaits the characterization of $n p r l 2$ and $n p r l 3$ null alleles.

Nprl2 and Nprl3 inhibit apoptosis in young egg chambers by downregulating TORC1 activity during nutrient stress. We reasoned that the young egg chamber death observed in nprl2 RNAi and nprl3 RNAi females resulted from the inability to downregulate TORC1 activity upon aminoacid starvation. The downregulation of TORC1 activity in young egg chambers likely triggers an adaptive response that renders young egg chambers resistant to nutrient stress. In order to test this model, we examined whether the TORC1 inhibitor rapamycin could rescue the starvation sensitivity of nprl2 RNAi and npr3 RNAi females. We found that treating females with rapamycin markedly decreased the percentage of young egg chambers that died under conditions of amino-acid restriction in the nprl2 RNAi and nprl3 RNAi background (Figures $4 \mathrm{a}-\mathrm{C}$ ). Furthermore, rapamycin-treated nprl2 RNAi and nprl3 RNAi females recover from amino-acid starvation with kinetics similar to those observed in control RNAi females (Figure 4d). Finally, rapamycin treatment did not trigger apoptosis in young egg chambers under fed conditions as has been reported for midstage egg chambers (Supplementary Figure S5d). ${ }^{8}$ These data indicate that the protective function of Nprl2/3 lies in its ability to downregulate TORC1 activity in response to aminoacid scarcity.

Consistent with the importance of lowering TORC1 activity during periods of nutrient stress, we determined that knocking
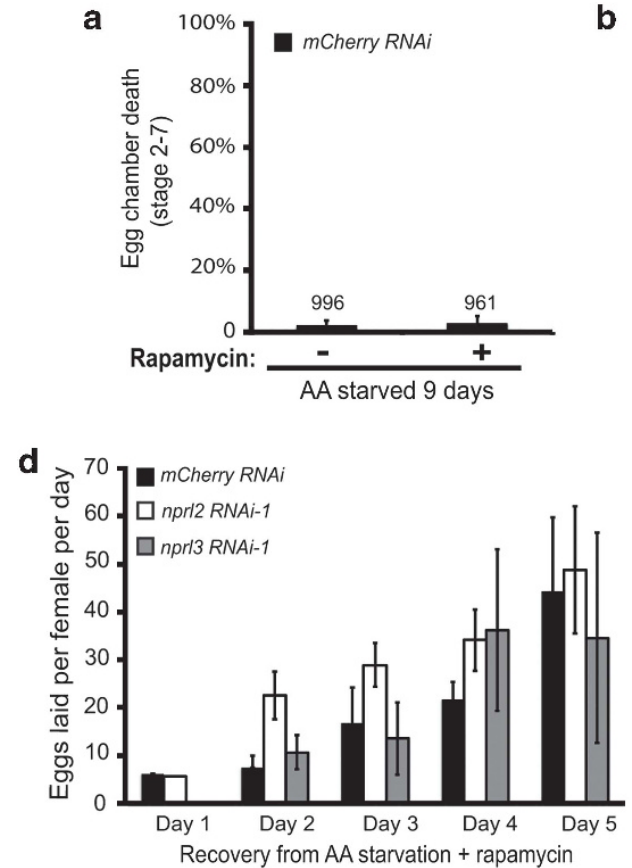
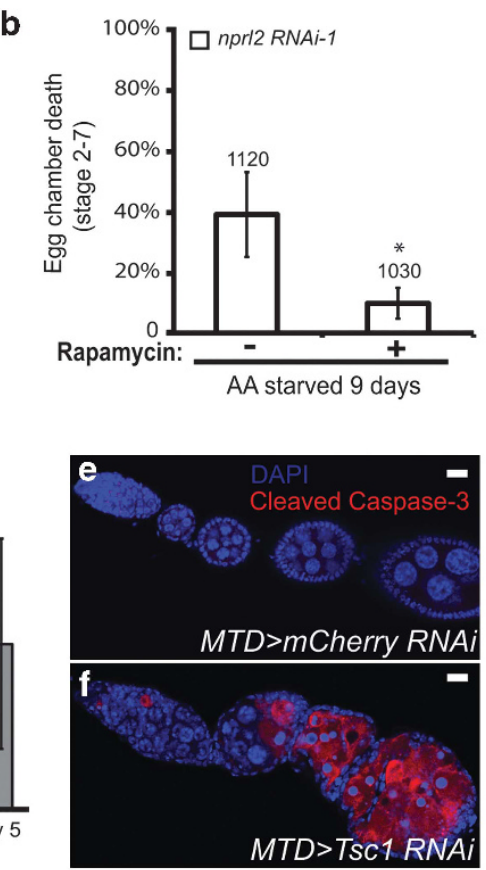
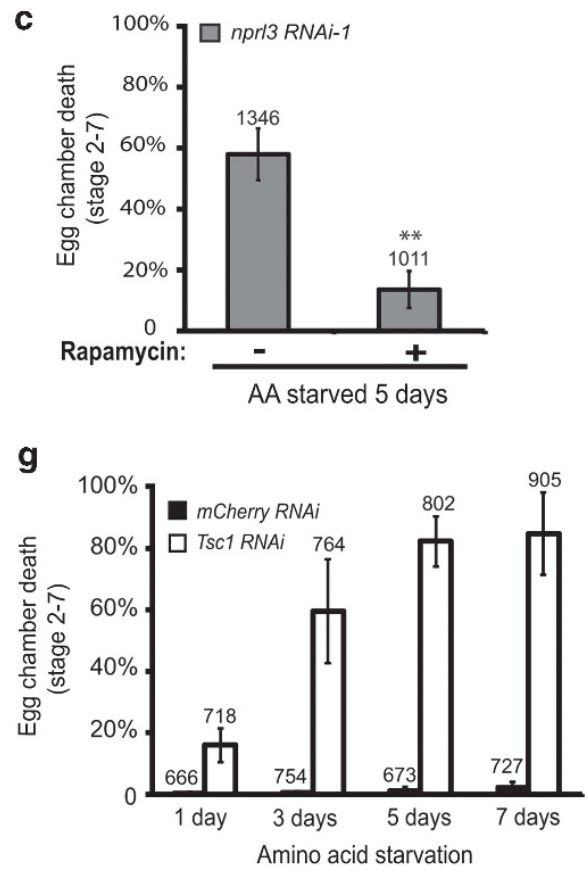

Figure 4 TORC1 inhibition promotes young egg chambers survival during amino-acid starvation. (a) MTD> mcherry RNAifemales were cultured on $20 \%$ sucrose plus or minus rapamycin for 9 days. (b) MTD > nprl2 RNAifemales were cultured on $20 \%$ sucrose plus or minus rapamycin for 9 days. (c) MTD $>$ nprl3 RNAifemales were cultured on $20 \%$ sucrose plus or minus rapamycin for 5 days. Data represent the percentages of dead or dying egg chambers (stage $2-7$ ). (d) Females were cultured on $20 \%$ sucrose plus rapamycin for 7 days and then transferred to fly media containing wet yeast. Data show eggs laid numbers after the cessation of starvation. (e, f) MTD $>m C h e r r y ~ R N A i$ and MTD > Tsc1 RNAifemales were cultered on $20 \%$ sucrose (AA-) for 5 days. The young egg chambers were labeled with DAPI (blue) and cleaved Caspase-3 (red). Bar, $10 \mu \mathrm{m}$. (f) MTD > Tsc1 RNAi females were cultured on $20 \%$ sucrose (AA-) for indicated days. Percentages of dead egg chambers (stage $2-7$ ) were recorded. Error bars represent S.D. ${ }^{\star} P<0.05$; ${ }^{* *} P<0.01$. In a-c and $\mathbf{g}$, the numbers above bars represent the number of young egg chambers (stages $2-7$ ) examined 
down the potent TORC1 inhibitor Tuberous sclerosis 1 (Tsc1) in the female germline also sensitized young egg chambers to amino-acid starvation (Figures $4 \mathrm{e}-\mathrm{g}$ ). The Tsc1/2 complex serves as a GTPase-activating protein (GAP) for the small GTPase Rheb, which functions as a positive regulator of TORC1 activity. ${ }^{37,38}$ In Drosophila, mutations in Tsc1 and Tsc2 (gigas) increase the baseline levels of TORC1 activity. ${ }^{39-42}$ Thus, our data suggest that the increased baseline level TORC1 activity observed in the Tsc1 RNAi knockdowns results in increased sensitivity to nutrient stress. Taken together, our data support the model that Nprl2 and Nprl3 act to inhibit TORC1 activity in response to amino-acid deprivation in Drosophila and that restricting TORC1 activity in the female germline is critical to the maintenance of fertility during times of protein scarcity.

Tsc1 acts downstream of Akt1 in the female germline. In the course of our studies on the role of TORC1 inhibitors in oogenesis, we examined the role of the serine threonine kinase Akt1. Akt1 regulates multiple pathways including, but not limited to, cell size and glucose metabolism. ${ }^{43}$ Many growth factors positively regulate TORC1 signaling through the Akt1-dependent phosphorylation and deactivation of Tsc2. ${ }^{4,45}$ However, recent data from Drosophila suggest that this relationship may not be relevant in vivo. ${ }^{46,47} \mathrm{We}$ found that knocking down Akt1 in germline cells using RNAi resulted in a block to oogenesis, with females containing extremely small ovaries (Figure $5 \mathrm{c}$ ). The small size of the Akt1 RNAi ovaries is consistent with RNAi knockdowns of the core TORC1 component raptor as well as a previous description of Tor-mutant ovaries ${ }^{6,48}$ (Figure $5 b$ ). Consistent with the Tsc1/2 complex being downstream of Akt1, coexpression of Akt1 RNAi and Tsc1 RNAi constructs strongly rescued the ovarian phenotype (Figure $5 \mathrm{~d}$ ). These data indicate that Akt1 regulates growth and development in the female germline at least in part by inhibiting the inappropriate activation of the Tsc1/2 complex. However, we note that our data do not address whether Tsc1/2 components are direct targets of Akt1 phosphorylation. Notably, coexpression of the nprl2 RNAi or nprl3 RNAi constructs fails to rescue the Akt1 RNAi phenotype (Figures $5 \mathrm{e}$ and f). Taken together our data strongly suggest that, as is observed in Schizosaccharomyces pombe, the Tsc1/2 and $\mathrm{Nprl2/3}$ complexes both negatively regulate TORC1 activity, but respond to different upstream signaling pathways. ${ }^{22}$

\section{Discussion}

In yeast Npr2 and Npr3 are components of an upstream pathway that inhibits TORC1 in response to amino-acid scarcity and are essential when cells are grown on a poor nitrogen source. ${ }^{21-23}$ Here we demonstrate that the Npr2 and Npr3 orthologs, Nprl2 and Nprl3, retain an essential role in response to amino-acid limitation in Drosophila. We show that Nprl2 and Nprl3 inhibit TORC1 signaling in the female germline in response to amino-acid starvation. Moreover, our data revealed that during early oogenesis the failure to downregulate TORC1 during periods of amino-acid stress triggers apoptosis. Thus, in the ovary Nprl2/3 exhibit an antiapoptotic function by preventing the inappropriate activation of TORC1 under conditions of amino-acid stress. Finally, we demonstrate that Nprl2/3 work in concert with the TORC1 inhibitors Tsc1/2 to fine tune TORC1 activity during oogenesis.

\section{Deregulated TORC1 activation sensitizes young egg} chambers to apoptosis. In wild-type females, amino-acid starvation triggers a near halt to growth and development in early-stage egg chambers., ${ }^{2,5}$ This inactive state can be maintained for long periods of time without these egg chambers degenerating. Protecting young egg chambers during periods of starvation helps females rapidly recover when a protein source becomes available. We have shown that when nprl2 or nprl3 is knocked down in the female
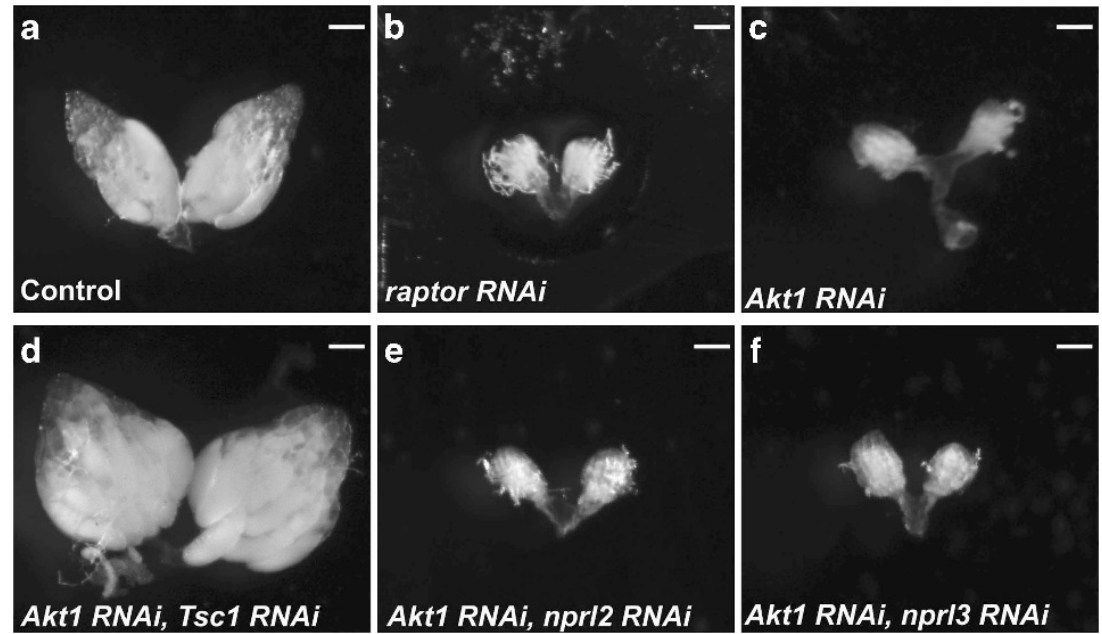

Figure 5 The TORC1 inhibitor Tsc1 is downstream of Akt1 in the female germline. (a) nanos-GAL4; UAS-mCherry RNAi, (b) nanos-GAL4; UAS-raptor RNAi, (c) nanosGAL4; UAS-Akt1 RNAi, (d) nanos-GAL4; UAS-Akt1 RNAi/UAS-Tsc1 RNAi, (e) nanos-GAL4; UAS-Akt1 RNAi/UAS-nprl2 RNAi and (f) nanos-GAL4; UAS-Akt1 RNAi/UASnprl3 RNAi flies were cultured on standard fly media with wet yeast 2 days before dissection. The germline-specific driver nanos-GAL4 was used to drive expression of the RNAi constructs. (a, d) Hundred percent of the ovaries examined contained mature (stage 13-14) egg chambers. (b, c, e, f) Zero percent of the ovaries examined contained egg chambers beyond stage 5: (a-f) $N>50$ ovaries. Bar, $50 \mu \mathrm{m}$ 


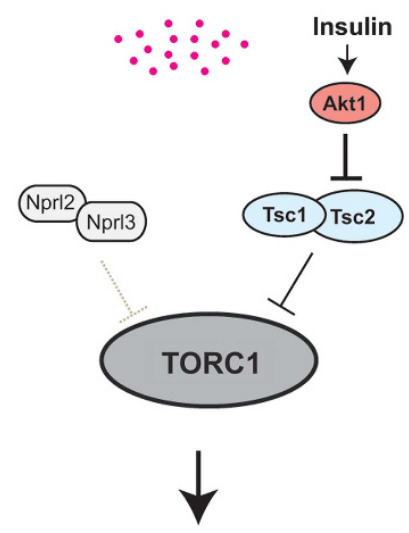

Growth

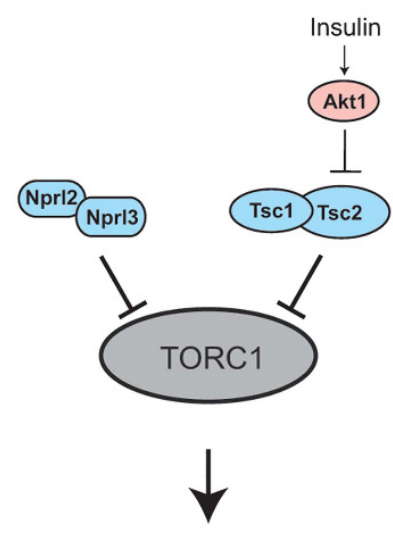

Stress response
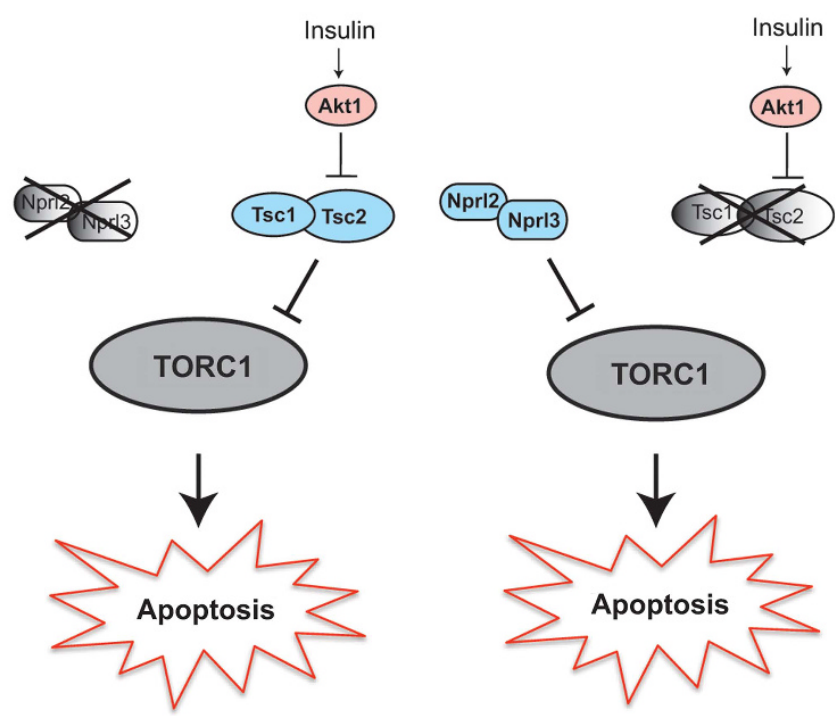

Figure 6 The TORC1 inhibitors Nprl2/3 and Tsc1/2 prevent apoptosis during amino-acid starvation in young egg chambers. (a) In the presence of amino acids, the TORC1 inhibitors Nprl2/3 are inactive while the insulin pathway inhibits the activity of Tsc1/2. Thus, TORC1 activity is high driving anabolic metabolism and growth. (b) In the absence of amino acids, Nprl2/3 functions to inhibit TORC1 activity. Amino-acid starvation also results in reduced insulin signaling, leading to activation of the Tsc1/2 complex due to the reduced activity of its inhibitor Akt1. Together Nprl2/3 and Tsc1/2 sufficiently inhibit TORC1 activity to induce an essential stress response (c) In nprl2 or $n p r l 3 \mathrm{knockdowns,}$ cells fail to adequately downregulate TORC1 activity in response to amino-acid starvation triggering apoptosis. (d) In Tsc1 knockdowns, TORC1 activity also remains inappropriately high during amino-acid starvation triggering apoptosis

germline, young egg chambers undergo apoptosis at high rates in response to amino-acid limitation. The TORC1 inhibitor rapamycin rescued this apoptotic phenotype. From these experiments we conclude that in young egg chambers the protective function of Nprl2/3 lies in its ability to inhibit TORC1 activity (Figure 6). Thus, in metazoans, which have evolved alternative pathways to regulate TORC1, the basic physiological requirement for $\mathrm{Nprl2} / \mathrm{Nprl3}$ in response to amino-acid starvation has been retained. This is the first in vivo demonstration of a metabolic requirement for Nprl2 and Nprl3 during amino-acid limitation in a metazoan.

In addition to Nprl2/3, we found that the Tsc1/2 complex is also required for the response to amino-acid stress during early oogenesis. The Tsc1/2 complex inhibits the small GTPase Rheb, which activates TORC1. ${ }^{37,38}$ As is observed in $n p l 2$ and nprl3 depletions, in Tsc1 germline depletions, young egg chambers undergo apoptosis in response to amino-acid starvation. In Drosophila, the insulin pathway controls ovarian cyst growth downstream of the Tor kinase. ${ }^{3,6}$ Moreover, it is well established in mammalian cells that insulin positively regulates TORC1 by activating the PI3K-Akt1 pathway, which inhibits Tsc2. ${ }^{44,45,49}$ Here we report that Tsc1 is a critical downstream effector of Akt1 in the female germline. Taken together, these data suggest the model that insulin signaling regulates TORC1 in early egg chambers upstream of both Akt1 and Tsc1/2 during nutrient stress.

In summary, our data indicate that Nprl2/3 and the Tsc1/2 complex are both required to inhibit TORC1 activity in response to amino-acid scarcity (Figure 6). Knocking out either the TORC1 inhibitory pathway renders young egg chambers sensitive to amino-acid starvation, because under conditions of cellular stress inappropriately high TORC1 activity triggers apoptosis (Figures $6 \mathrm{c}$ and d). A similar phenomenon has been documented in mammals where an increase in TORC1 activity observed in $\mathrm{TsC1}^{-/-}$and $\mathrm{TsC2}^{-1-}$ mutants sensitizes cells to apoptosis in response to multiple cellular stresses including energy status and DNA damage. ${ }^{27,50,51}$ We predict that in many tissues these two independent TORC1 inhibitory pathways, Tsc1/2 and Nprl2/3, will work in concert to fine tune TORC1 activity in response to various developmental and environmental inputs.

Tsc1 is a critical downstream effector of Akt in the female germline. Akt1 integrates inputs from multiple upstream signaling pathways and positively influences cell size and anabolic metabolism. We found that knocking down Akt1 in the female germline resulted in a severe block to oogenesis (Figure 5c). Notably, the coexpression of Akt1 RNAi and Tsc1 RNAi constructs rescued the Akt1 RNAi ovarian phenotype (Figure $5 \mathrm{~d}$ ). These data strongly suggest that Akt1 regulates growth and development in the female germline at least in part by inhibiting the activation of the Tsc1/2 complex. However, we note that our data do not address whether the Tsc1 or Tsc2 proteins are direct targets for Akt1 phosphorylation. Indeed, previous work found that in Drosophila deleting the Akt1 phosphorylation consensus sites on Tsc2 and Tsc1 had no physiological consequences when examined in vivo. ${ }^{46,47}$ Thus, our data suggest that either Akt1 phosphorylates and inhibits Tsc1 and/or Tsc2 at a previously unrecognized site, or, alternatively, in the female germline the inhibition of the Tsc $1 / 2$ complex by Akt 1 is indirect. Further experiments will be required to distinguish between these two models. We also examined whether Nprl2 and Nprl3 function as critical downstream targets of Akt1. 
However, coexpression of the npr2 RNAi or npr3 RNAi constructs failed to rescue any aspect of the Akt1 RNAi ovarian phenotype (Figures $5 \mathrm{e}$ and f). These data suggest that Nprl2 and Nprl3 are unlikely to be important downstream effectors of $A k t 1$ in the ovary.

\section{Materials and Methods}

Drosophila strains and genetics. MTD-GAL4 $\quad(\mathrm{P}\{\mathrm{W}[+\mathrm{mC}]=$ otu-GAL4::VP16.R $\left.\} 1, \quad w{ }^{*}\right] ; P\{w[+m C]=G A L 4-n o s . N G T\} 40 ; \quad P\{w[+m C]=$ GAL4::VP16-nos.UTR\}CG6325[MVD1]), ${ }^{52}$ UAS-Tsc1 RNAi (y ${ }^{1} c^{*} v^{1}$; P\{TRiP.GL00012\}attP2), UAS-mCherry RNAi $\left(y^{1} \mathrm{sc}^{*} \mathrm{v}^{1}\right.$; P\{VALIUM20-mCherry\} attP2), UAS-Akt1 RNAi $\left(y^{1} v^{1}\right.$; P\{TRiP.HMS00007\}attP2), UAS-Raptor RNAi $\left(y^{1} \quad\right.$ sc $^{*} v^{1} ;$ P\{TRiP.HMS00124\}attP2) and UASp-mCherry-Atg8a ${ }^{53}\left(y^{1} w^{1118}\right.$ P\{UASp-mCherry-Atg8a\}2; $\mathrm{Dr}^{1} / \mathrm{TM} 3, \mathrm{Ser}^{1}$ ) lines were obtained from the Bloomington Stock Center. Tub-GFP-LAMP1 ( $w^{1118} ; \mathrm{P}\{\mathrm{W}+$, Tub $>$ GFP. LAMP\} $/$ CyO; TM6b, Hu boss ${ }^{1} / \mathrm{Sb}$ boss $\left.{ }^{1}\right)^{30}$ was kindly provided by Helmut Kramer (UT Southwestern). Nanos-Gal4 (P\{NANOS GAL4 VP-16\}, yw; D/TM3, Ser, Sb) ${ }^{54}$ was kindly provided by Sharon Bickel (Dartmouth College). The generation of the UAS-Tsc1 RNAi, UAS-mCherry RNAi and UAS-Raptor RNAi lines are described in $\mathrm{Ni}$ et $\mathrm{al} .^{34}$

All fly stocks were maintained on JAZZ-mix Drosophila food (Fisher Scientific, Waltham, MA, USA) at $25^{\circ} \mathrm{C}$. For amino-acid starvation, flies were cultured on media consisting of phosphate-buffered saline (PBS), $20 \%$ sucrose and $0.8 \%$ agarose. Rapamycin treatment was preformed as previously reported. ${ }^{55}$ In brief, rapamycin (LC Laboratories, Woburn, MA, USA) was added to food for a final concentration of $200 \mu \mathrm{M}$. To measure egg production, four pairs of male and female flies were cultured on grape juice media (Genesee Scientific, San Diego, CA, USA) together with wet yeast. The number of eggs laid was counted every $24 \mathrm{~h}$.

Plasmids and dsRNA. The nprl2 and $n p r l 3$ coding regions were amplified using specific primers (Supplementary Table S2) and then inserted into a pENTR$1 \mathrm{~A}$ vector (Invitrogen, Carlsbad, CA, USA). The pENTR-Nprl2 plasmid was recombined into pAHW (DGRC) and pPGW vectors (DGRC) to generate Act-HANpr2 and UASp-GFP-Nprl2 plasmids using Gateway LR Clonase II Enzyme (Invitrogen). The pENTR-Nprl3 plasmid was inserted with mCherry coding region at the $\mathrm{C}$ terminal of Nprl3 and then recombined into a pPW vector (DGRC) to generate UASp-Nprl3-mCherry. The nprl3 coding region was amplified and inserted into a pAC5.1-His-V5 vector (Invitrogen) to construct the Act-Nprl3-His-V5 plasmid.

To generate double-stranded RNA, DNA templates were PCR amplified to include a $5^{\prime} T 7$ RNA polymerase binding site. PCR products were purified and used as templates to produce dsRNA using the MEGAscript RNAi kit (Ambion, Austin, TX, USA). Primer sequences used to generate nprl2, nprl3 and Tsc1 DNA templates for synthesizing double-strand RNA (dsRNA) are listed in Supplementary Table S3.

Generation of transgenic lines. The UASp-GFP-Nprl2 and UASp-Nprl3$m C h e r r y$ plasmids were used to generate transgenic lines (Best Gene Inc., Chino Hills, CA, USA).

Short hairpin RNA sequences targeting the nprl2 or nprl3 transcripts (Supplementary Table S4) were inserted into the Valium 20 or Valium 22 vectors. ${ }^{34}$ The plasmids were injected into $y^{1} s c^{1} v^{1} ; P\{y[+t 7.7]=$ CaryP $\}$ attP2 flies to generate transgenic lines (Genetic Service Inc., Cambridge, MA, USA).

Cell culture. Drosophila S2 cells were cultured at $26^{\circ} \mathrm{C}$ in Schneider's medium (Invitrogen) supplemented with 10\% fetal bovine serum (FBS, Invitrogen), $50 \mathrm{U} / \mathrm{ml}$ penicillin and $50 \mu \mathrm{g} / \mathrm{ml}$ streptomycin. For amino-acid starvation, cells were cultured in amino-acid-free Schneider's medium (US Biological, Swampscott, MA USA). RNA interference in S2 cells was performed as described previously. ${ }^{56}$

S2 cell size analysis was determined as described in Cully and Downward. ${ }^{57}$ In brief, S2 cells were fixed and stained with propidium iodide, and then were detected using a flow cytometry (FACSCalibur, BD Bioscience, San Jose, CA, USA). The mean value of forward light scatter (FSC) in the gated G1 phase was used to determine cell size.

S2 cell transfection, immunopreciptation (IP) and western blot analysis. For transfection, S2 cells grown in six-well plates were transfected with $0.4 \mu \mathrm{g}$ plasmids using Effectene Transfection Reagent (Qiagen, Hilden,
Germany). Three days later, the S2 cells were lysed using $1 \mathrm{ml}$ IP buffer ( $1 \%$ NP40, PBS) containing complete protease inhibitors (Roche, Mannheim, Germany). Cell lysates were centrifuged and the supernatants were collected. For IPs, the protein extracts were incubated with $3 \mu \mathrm{g}$ anti-V5 antibody (Invitrogen), $30 \mu \mathrm{l}$ protein G agarose (Millipore, Billerica, MA, USA) and $10 \mu \mathrm{l}$ protein $A$ agarose (Roche) overnight at $4{ }^{\circ} \mathrm{C}$. The agarose beads were collected and washed six times with IP buffer, and then detected by western blot.

Antibodies were used at the following concentrations for western blot: mouse anti- $\alpha$-tubulin at 1:50000 (Jackson ImmunoResearch, West Grove, PA, USA), mouse anti-V5 antibody at 1: 4000 (Invitrogen), rabbit anti-P-4E-BP at 1: 1000 (Cell Signaling Technology, Danvers, MA, USA), rat anti-HA antibody at 1: 1000 (Roche) and rat anti-4E-BP at $1: 3000 .^{58}$

Immunofluorescence and live cell imaging. Immunofluorescence was performed as described in Hong et al., ${ }^{59}$ using a rabbit anti-cleaved Caspase 3 ( $1: 200$, Cell Signaling Technology) antibody. Secondary antibody was anti-rabbitconjugated to Alexa Fluor 594 (Invitrogen) and used at 1:1000. Nuclei were visualized by staining the DNA with DAPI (Invitrogen). Dead egg chambers were indentified by condensed DNA staining (pyknotic nuclei). Images were acquired using an Olympus FV1000 confocal microscope (Olympus, Tokyo, Japan).

Live cells images were obtained from freshly dissected ovaries. For LysoTracker staining, tissues were incubated with LysoTracker (1:5000, Invitrogen) and Hoechst (1:10000, Invitrogen) for $5 \mathrm{~min}$, and then washed and mounted. Ovaries from fed females were dissected, washed and mounted in Schneider's medium, while the Ovaries from starved females were dissected, washed and mounted in PBS. Images were acquired using an Olympus FV1000 confocal microscope.

\section{Conflict of Interest}

The authors declare no conflict of interest.

Acknowledgements. We thank members of the Lilly laboratory for comments on the manuscript. We thank the TRiP at Harvard Medical School (NIH/NIGMS R01-GM084947) for providing transgenic RNAi plasmid vectors used in this study. We thank Aurelio Teleman, Sharon Bickel and Helmut Kramer for providing reagents. This work was supported by the Intramural Program of the Eunice Kennedy Shriver National Institute of Child Health and Human Development, NIH.

1. King RC. The meiotic behavior of the Drosophila oocyte. Int Rev Cytol 1970; 28: $125-168$

2. Drummond-Barbosa D, Spradling AC. Stem cells and their progeny respond to nutritional changes during Drosophila oogenesis. Dev Biol 2001; 231: 265-278.

3. LaFever L, Drummond-Barbosa D. Direct control of germline stem cell division and cyst growth by neural insulin in Drosophila. Science 2005; 309: 1071-1073.

4. Spradling AC. In: Bate M, Martinez-Arias A (eds). The Development of Drosophila melanogaster. Cold Spring Harbor Laboratory Press: NY, USA, 1993, pp 1-70.

5. Shimada Y, Burn KM, Niwa R, Cooley L. Reversible response of protein localization and microtubule organization to nutrient stress during Drosophila early oogenesis. Dev Biol 2011; 355: 250-262.

6. LaFever L, Feoktistov A, Hsu HJ, Drummond-Barbosa D. Specific roles of Target of rapamycin in the control of stem cells and their progeny in the Drosophila ovary. Development 2010; 137: 2117-2126.

7. Pritchett TL, Tanner EA, McCall K. Cracking open cell death in the Drosophila ovary. Apoptosis 2009; 14: 969-979.

8. Thomson TC, Fitzpatrick KE, Johnson J. Intrinsic and extrinsic mechanisms of oocyte loss. Mol Hum Reprod 2010; 16: 916-927.

9. Sun P, Quan Z, Zhang B, Wu T, Xi R. TSC1/2 tumour suppressor complex maintains Drosophila germline stem cells by preventing differentiation. Development 2010; 137 : 2461-2469

10. Pritchett TL, McCall K. Role of the insulin/Tor signaling network in starvation-induced programmed cell death in Drosophila oogenesis. Cell Death Differ 2012; 19: 1069-1079.

11. Barth JM, Szabad J, Hafen E, Kohler K. Autophagy in Drosophila ovaries is induced by starvation and is required for oogenesis. Cell Death Differ 2011; 18: 915-924.

12. Zhang H, Stallock JP, Ng JC, Reinhard C, Neufeld TP. Regulation of cellular growth by the Drosophila target of rapamycin dTOR. Genes Dev 2000; 14: 2712-2724.

13. Efeyan A, Zoncu R, Chang S, Gumper I, Snitkin H, Wolfson RL et al. Regulation of mTORC 1 by the Rag GTPases is necessary for neonatal autophagy and survival. Nature 2013; 493: 679-683.

14. Neufeld TP. Genetic analysis of TOR signaling in Drosophila. Curr Top Microbiol Immunol 2004; 279: 139-152. 
15. Kim DH, Sarbassov DD, Ali SM, King JE, Latek RR, Erdjument-Bromage $\mathrm{H}$ et al. mTOR interacts with raptor to form a nutrient-sensitive complex that signals to the cell growth machinery. Cell 2002; 110: 163-175.

16. Jewell JL, Guan KL. Nutrient signaling to mTOR and cell growth. Trends Biochem Sci2013; 38: 233-242.

17. Sancak Y, Bar-Peled L, Zoncu R, Markhard AL, Nada S, Sabatini DM. Ragulator-Rag complex targets mTORC1 to the lysosomal surface and is necessary for its activation by amino acids. Cell 2010; 141: 290-303.

18. Kim E, Goraksha-Hicks P, Li L, Neufeld TP, Guan KL. Regulation of TORC1 by Rag GTPases in nutrient response. Nat Cell Biol 2008; 10: 935-945.

19. Laplante M, Sabatini DM. mTOR signaling in growth control and disease. Cell 2012; 149: 274-293.

20. Rabinowitz JD, White E. Autophagy and metabolism. Science 2010; 330: 1344-1348.

21. Neklesa TK, Davis RW. A genome-wide screen for regulators of TORC1 in response to amino acid starvation reveals a conserved Npr2/3 complex. PLoS Genet 2009; 5: e1000515.

22. Ma N, Liu Q, Zhang L, Henske EP, Ma Y. TORC1 signaling is governed by two negative regulators in fission yeast. Genetics 2013; 195: 457-468.

23. Panchaud N, Peli-Gulli MP, De Virgilio C. Amino acid deprivation inhibits TORC1 through a GTPase-activating protein complex for the Rag family GTPase Gtr1. Sci. Signal 2013; 6: ra42.

24. Bar-Peled L, Chantranupong L, Cherniack AD, Chen WW, Ottina KA, Grabiner BC et al. A Tumor suppressor complex with GAP activity for the Rag GTPases that signal amino acid sufficiency to mTORC1. Science 2013; 340: 1100-1106.

25. Ueda K, Kawashima H, Ohtani S, Deng WG, Ravoori M, Bankson J et al. The 3p21.3 tumor suppressor NPRL2 plays an important role in cisplatin-induced resistance in human non-small-cell lung cancer cells. Cancer Res 2006; 66: 9682-9690.

26. Wu X, Tu BP. Selective regulation of autophagy by the Iml1-Npr2-Npr3 complex in the absence of nitrogen starvation. Mol Biol Cell 2011; 22: 4124-4133.

27. Inoki K, Zhu T, Guan KL. TSC2 mediates cellular energy response to control cell growth and survival. Cell 2003; 115: 577-590.

28. Binda M, Péli-Gulli MP, Bonfils G, Panchaud N, Urban J, Sturgill TW et al. The Vam6 GEF controls TORC1 by activating the EGO complex. Mol Cell 2009; 35: 563-573.

29. Nezis IP, Lamark T, Velentzas AD, Rusten TE, Bjørkøy G, Johansen T et al. Cell death during Drosophila melanogaster early oogenesis is mediated through autophagy. Autophagy 2009; 5: 298-302.

30. Akbar MA, Ray S, Kramer H. The SM protein Car/Vps33A regulates SNARE-mediated trafficking to lysosomes and lysosome-related organelles. Mol Biol Cell 2009; 20: 1705-1714.

31. Dokudovskaya S, Waharte F, Schlessinger A, Pieper U, Devos DP, Cristea IM et al. A conserved coatomer-related complex containing Sec13 and Seh1 dynamically associates with the vacuole in Saccharomyces cerevisiae. Mol Cell Proteomics 2011; 10: M110. 006478

32. Enyenihi AH, Saunders WS. Large-scale functional genomic analysis of sporulation and meiosis in Saccharomyces cerevisiae. Genetics 2003; 163: 47-54.

33. Spielewoy N, Guaderrama M, Wohlschlegel JA, Ashe M, Yates JR 3rd, Wittenberg C. Npr2, yeast homolog of the human tumor suppressor NPRL2, is a target of Grr1 required for adaptation to growth on diverse nitrogen sources. Eukaryotic Cell 2010; 9: 592-601.

34. Ni JQ, Zhou R, Czech B, Liu LP, Holderbaum L, Yang-Zhou D et al. A genome-scale shRNA resource for transgenic RNAi in Drosophila. Nat Methods 2010; 8: 405-407.

35. Fan Y, Bergmann A. The cleaved-Caspase-3 antibody is a marker of Caspase-9-like DRONC activity in Drosophila. Cell Death Differ 2010; 17: 534-539.

36. Jenkins VK, Timmons AK, McCall K. Diversity of cell death pathways: insight from the fly ovary. Trends Cell Biol 2013; 23: 567-574.

37. Inoki K, Li Y, Xu T, Guan KL. Rheb GTPase is a direct target of TSC2 GAP activity and regulates mTOR signaling. Genes Dev 2003; 17: 1829-1834.
38. Zhang Y, Gao X, Saucedo LJ, Ru B, Edgar BA, Pan D. Rheb is a direct target of the tuberous sclerosis tumour suppressor proteins. Nat Cell Biol 2003; 5: 578-581.

39. Ito N, Rubin G. M. gigas, a Drosophila homolog of tuberous sclerosis gene product-2, regulates the cell cycle. Cell 1999; 96: 529-539.

40. Tapon N, Ito N, Dickson BJ, Treisman JE, Hariharan IK. The Drosophila tuberous sclerosis complex gene homologs restrict cell growth and cell proliferation. Cell 2001; 105: 345-355.

41. Gao X, Zhang Y, Arrazola P, Hino O, Kobayashi T, Yeung RS et al. Tsc tumour suppressor proteins antagonize amino-acid-TOR signalling. Nat Cell Biol 2002; 4: 699-704.

42. Radimerski T, Montagne J, Hemmings-Mieszczak M, Thomas G. Lethality of Drosophila lacking TSC tumor suppressor function rescued by reducing dS6K signaling. Genes Dev 2002; 16: 2627-2632.

43. Scanga SE, Ruel L, Binari RC, Snow B, Stambolic V, Bouchard D et al. The conserved $\mathrm{PI}$ 'K/PTEN/Akt signaling pathway regulates both cell size and survival in Drosophila. Oncogene 2000; 19: 3971-3977.

44. Gao X, Pan D. TSC1 and TSC2 tumor suppressors antagonize insulin signaling in cell growth. Genes Dev 2001; 15: 1383-1392.

45. Inoki K, Li Y, Zhu T, Wu J, Guan KL. TSC2 is phosphorylated and inhibited by Akt and suppresses mTOR signalling. Nat Cell Biol 2002; 4: 648-657.

46. Dong J, Pan D. Tsc2 is not a critical target of Akt during normal Drosophila development. Genes Dev 2004; 18: 2479-2484.

47. Schleich S, Teleman AA. Akt phosphorylates both Tsc1 and Tsc2 in Drosophila, but neither phosphorylation is required for normal animal growth. PLoS one 2009; 4: e6305.

48. Zhang $Y$, Billington $\mathrm{CJ} J \mathrm{~J}$, Pan D, Neufeld TP. Drosophila target of rapamycin kinase functions as a multimer. Genetics 2006; 172: 355-362.

49. Manning BD, Cantley LC. AKT/PKB signaling: navigating downstream. Cell 2007; 129 1261-1274.

50. Shaw RJ, Bardeesy N, Manning BD, Lopez L, Kosmatka M, DePinho RA et al. The LKB1 tumor suppressor negatively regulates mTOR signaling. Cancer Cell 2004; 6 : 91-99.

51. Lee $\mathrm{CH}$, Inoki K, Karbowniczek M, Petroulakis E, Sonenberg N, Henske EP et al. Constitutive mTOR activation in TSC mutants sensitizes cells to energy starvation and genomic damage via p53. Embo J 2007; 26: 4812-4823.

52. Petrella LN, Smith-Leiker T, Cooley L. The Ovhts polyprotein is cleaved to produce fusome and ring canal proteins required for Drosophila oogenesis. Development 2007; 134: 703-712.

53. Nezis IP, Stravopodis DJ, Margaritis LH, Papassideri IS. Autophagy is required for the degeneration of the ovarian follicular epithelium in higher Diptera. Autophagy 2006; 2 : 297-298.

54. Van Doren M, Williamson AL, Lehmann R. Regulation of zygotic gene expression in Drosophila primordial germ cells. Curr Biol 1998; 8: 243-246.

55. Bjedov I, Toivonen JM, Kerr F, Slack C, Jacobson J, Foley A et al. Mechanisms of life span extension by rapamycin in the fruit fly Drosophila melanogaster. Cell Metab 2010; 11: 35-46.

56. Senger S, Csokmay J, lida-Jones T, Sengupta P, Lilly MA. The nucleoporin Seh1 forms a complex with Mio and serves an essential tissue specific function in Drosophila oogenesis. Development 2011; 138: 2133-2142.

57. Cully M, Genevet A, Warne P, Treins C, Liu T, Bastien J et al. A role for p38 stress-activated protein kinase in regulation of cell growth via TORC1. Mol Cell Biol 2010; 30: $481-495$.

58. Hahn K, Miranda M, Francis VA, Vendrell J, Zorzano A, Teleman AA. PP2A regulatory subunit PP2A-B' counteracts S6K phosphorylation. Cell Metab 2010; 11: 438-444.

59. Hong A, Lee-Kong S, lida T, Sugimura I, Lilly MA. The p27cip/kip ortholog dacapo maintains the Drosophila oocyte in prophase of meiosis I. Development 2003; 130 $1235-1242$.

Supplementary Information accompanies this paper on Cell Death and Differentiation website (http://www.nature.com/cdd) 